\title{
Improvements on Grasping the Object with Irregular Shapes
}

\author{
Alina Rodica Spanu ${ }^{1, *}$, George Dragoi ${ }^{2}$, and Iolanda Panait ${ }^{3}$ \\ ${ }^{1}$ University Politehnica of Bucharest, Mechatronics and Precision Engineering Department, 313 \\ Splaiul Independentei, Bucharest, Romania \\ ${ }^{2}$ University Politehnica of Bucharest, Foreign Languages Engineering Department, 313 Splaiul \\ Independentei, Bucharest, Romania \\ ${ }^{3}$ University Politehnica of Bucharest, Industrial Process Equipment Department, 313 Splaiul \\ Independentei, Bucharest, Romania
}

\begin{abstract}
The image processing technique has been rapidly developed during the last years due to the improvement of computer science and applied algorithms. The paper aims to analyse the method for designing the active surfaces of the gripper suitable for the object with irregular shapes. Concerning these particularities, we have taken the object picture in order to process it. By using the appropriate algorithms, the results were transferred in the three dimensional modelling software, so that the fingers were designed according to the object shapes. Finally, they have been manufactured using the $3 \mathrm{D}$ printer and the technical performances were analysed.
\end{abstract}

\section{Introduction}

Due to their important functional performances as final element of the robot, the grippers should be designed very accurately as they have to manipulate the object. There are large variety of plane and spatial mechanisms typically used as constructive solutions, as well as many possibilities of actuation taking into account the primary energy source, their accuracy and the grasping force range.

Regarding the working object, some classifications were done taking into account their external shape and the number of contact points in order to achieve the imposed stability level. As the theoretical and practice activities require, a connection between the number of contact points with the object and the force magnitude is applied.

When the external shape of the object does not fit the categories of surfaces defined by analytical relations, especially for their basic curves, the ideas presented before become more complex. Following this aspect, we have to be very carefully in choosing the position and the number of contact points chosen as future working points. The most important requirements are regarding the point position in front of the object, characterised by its planar or spatial coordinates inside the robot working space, and the optimum number of

\footnotetext{
* Corresponding author: alina.spanu@upb.ro
} 
these working points. Due to the irregular shape, an increasing number of working points correctly positioned according to surface curvature could influence the grasping performance and especially the accuracy.

The paper [1] deals with the synthesis method, which could be used for grasping with the advantages of closed loop actuated by servomotors. The image processing overcomes limitations current deep-learning grasping techniques by avoiding discrete sampling and long computation times. The paper [2] concerns with the novel algorithm for performing the grasping by edge detection particularly for ellipses. At first, the edge curvature is analysed because of splitting the curves into arcs. Then, based on geometry constraints the arcs are grouped into different quadrants ellipses. The algorithm is applied to real-time manipulation of objects for arranging and tracking purposes. The paper [3] presents an automated system that is able to track and grasp a moving object using images acquired with a Microsoft Kinetic sensor. The paper [4] points out the important aspect of cluster and its modularity in order to computer image processing. The described techniques is applied for agricultural researches in order to improve the determination of grain number during only few seconds. The container with the grain is placed beneath the camera and it uses an image processing device. The images are analysed in order to detect the edges of the objects and after that by using the Python code the counting of labelled images is provided. The paper [5] describes some algorithms used for boosting the performance and energy efficiency in digital image processing task based on mapping the data to the assigned memory cells and process the data within these cells. The paper [6] presents the method of compression and edge detection based on two-dimensional Markov-chain, which allows the image processing of more than 24 bits on pixel. The paper [7] analyses the advantages and disadvantages of image segmentation techniques as a procedure, which split a picture, which are comparative in some viewpoints being the primary step. There are numerous segmentation algorithms available in the literature, so that the result picture accuracy will be definitely.

This paper aims to point out the way we may determine the parameters of the active surfaces of the grasped objects, having irregular external shapes, by using camera for taking pictures that have to be processed later using appropriate software. For this experimental set-up we have used the MATLAB functions. As the result of this working stage, we may obtain the coordinates of points lying on the curves that defined the working surfaces, so the user has to choose these curves as they have an important role during the grasping. This method for establishing the coordinates by dimensional modelling could provide the possibility of numerical interpolation, so the mathematical equation of curves or surfaces could be written. By using the design with the three-dimensional software, two fingers of the gripper were computed, and the prototype was manufactured with the $3 \mathrm{D}$ - printer technique.

\section{The processing of working object image}

There are many technical fields where we may use the image processing, such as microscopy slides, computed tomography, robotics and agriculture area especially for harvesting for recording current conditions in the observed production or for research activities. Almost all of them have the goal of object edge detection with higher accuracy.

The object image gives us the most important necessary information about the object shape that will be considered as working external surface with its future contact points the user is interested in. Later, the designer has to choose correctly the final number of these points, so that the stability and the grasping force have to be achieved simultaneously.

As experimental set-up we have chosen a mouse with external irregular surfaces. We have taken the picture using the Microsoft LifeCam connected to the PC. The file has been 
saved in the Matlab working directory where the future MATLAB file will be saved too. The image was saved as Portable Network Graphics (.png). In order to read such file, we have used the function imread from the specialized library Image Processing [8,9], whose final result will be the data about the RGB image. The picture of our object is presented in Figure 1.

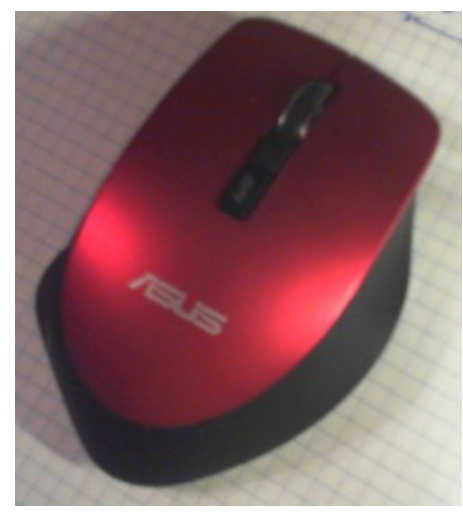

Fig. 1. The image taken with Microsoft LifeCam Fig. 2. The image tool with the coordinate values as .png file

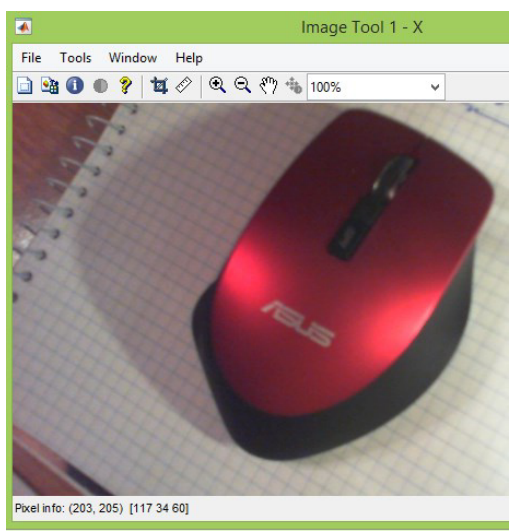

for the pixel.

By using the function imtool we may read the (X, Y) coordinates of each pixel of the image, so the values are displayed in the lower left corner of the picture. The Figure 2 gives the coordinates written in the lower left corner for the point where the mouse was put over.

The next function we have used is imshow, so we may visualise the object with its own colours using the RGB matrix. Next we have to establish the working area of this image by choosing the limits for cropping it. In our experimental set-up, we have learned the

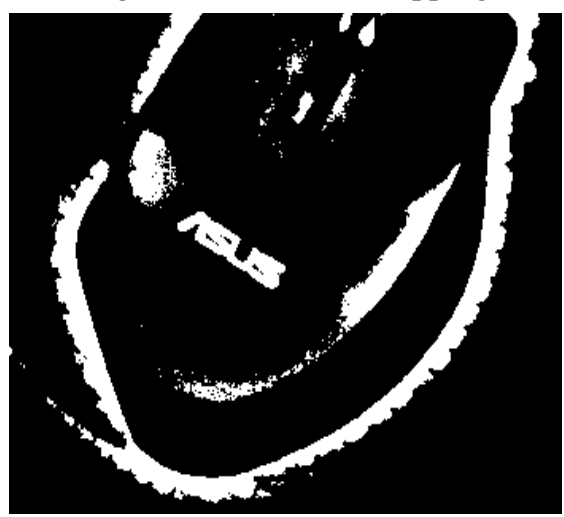

Fig. 3. The processed grey scale image. coordinates of upper left corner at first. We will pass over the image starting from this point going to the right along $\mathrm{X}$ direction and going down along Y direction.

Once we have delimited the area of interest we will convert the image into a grey scale by eliminating the hue and saturation information, while retaining the luminance. This grey scale image will be binary coded by using the function imbinarize. In such a way it will be used the local first - order image statistics around each pixel. A threshold is computed for each pixel using the local mean intensity around the neighbourhood of the pixel. The result of the grey scale image is presented in Figure 3.

We expect the result of the normalized image in the range $[0,1]$. Finally, we have obtained an array with 281 rows and 350 columns.

\section{The analyze of curves with future working points}

The aim of this working stage is to obtain the curves that have to be the boundaries of the object, so the working points could be found along them. In order to accomplish this task, 
we have to establish the starting point very carefully, because of accuracy increasing reasons. Starting from this point, there will be traced the outlines of the object as it may be found in the binary image obtained before. The nonzero pixels belong to the object and the zero pixels will indicate the background of the image.

Consequently, the function named bwtraceboundary has been used and one of its parameter is the searching direction set by the user. For instance, in our experimental set-up we have set the directions south, so the parameter is ' $\mathrm{S}$ ' (meaning south) for the $\mathrm{Y}$ coordinate. For the $\mathrm{X}$ coordinate range, we have used east direction, so the parameter is ' $\mathrm{E}$ '.
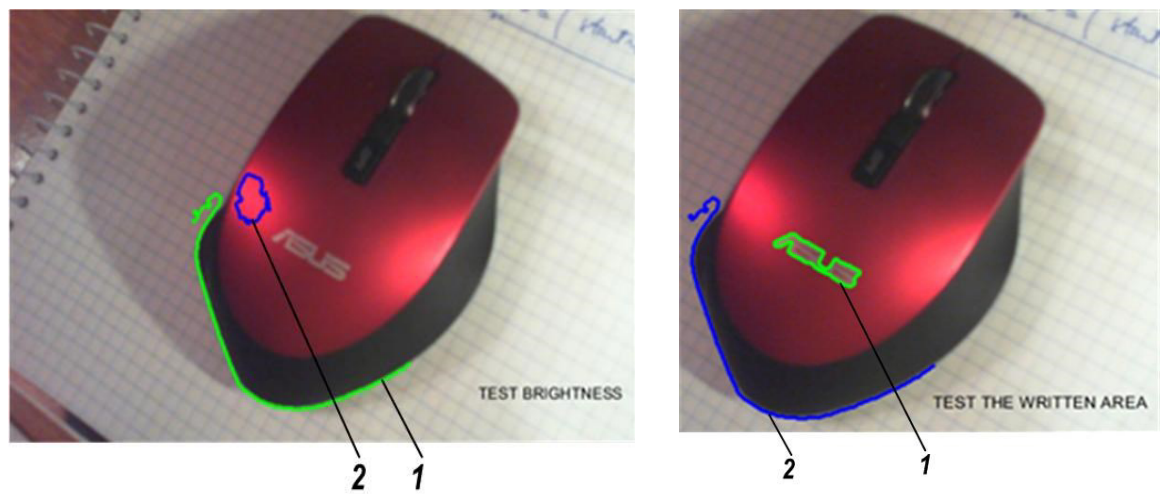

Fig. 4. The curve around the brightness area over the external surface (1) and the curve around the external boundary (2).
Fig. 5. The curve around the written area over the external surface (1) and the curve around the external boundary (2).

The results will be discussed below. The first case was developed in order to test the brightness along the external surface of the object, so the plotted curve was around the white area on this surface as it is shown in the Figure 3.

The curve 1 (Fig. 4.) was traced along the boundary of the external surface. Following the same goal, we have extended the searched area on the external surface of the object, so that we have delimited the area where some words have been written, based on the greyscale image. The results are pointed out in Figure 5. The next target was the process of finding out the curve plotted for the entire boundary of the external surface, so that we have

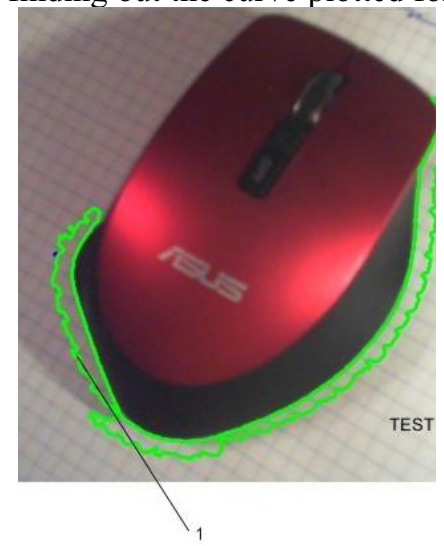
increased the number of tested pixels for both lateral boundaries and the final values are 1350 .

The result is presented in figure 6 where the entire curve 1 was plotted following the contrast of the binary image as it is presented in Figure 3.

As we may infer from this picture, the plotted green curve is following the area with the best contrast whiteblack around the object boundary. The plotted coordinates give all the points of this smooth curve inside, so they may be used for positioning the active surface of the gripper.

Fig. 6. The curve around the boundary of the external surface (green colour) based on the grey-scale image. 


\section{The experimental set-up}

Based on the computed results before, we have made the three dimensional model of the spline curve following the point coordinates by using CATIA V5 - R19. The surface lying on this curve was modeled with Generative Shape Design and after that the Part Body was

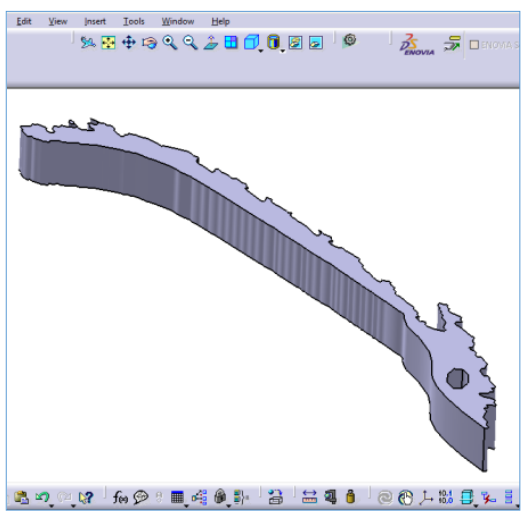

Fig. 7. The three dimensional model of the finger. obtained by thicken the surface. The Figure 7 presents the modeled part used as finger, so we may infer that it is corresponding closely with the processed grey scale image presented in Figure 3 and the active surface is lying on the spatial curve determining the position of contact points with the object.

The experimental set-up is presented in Figure 8. In order to prove the accuracy of the contact between the object with irregular active surfaces for grasping and the two designed fingers, we have printed them considered as virtual prototype [10]. Meantime, the theoretical study provided the two curves, which are manufactured, so we have use the electrical stepper motor 1 for positioning the object inside the both fingers with the goal of centering them. The movement direction should be analyzed

theoretically and practically, because of requirement regarding the using of central axis of the object.

The electrical motor is controlled with Arduino and the driver is Easy Driver. We have determined experimentally the translational axis of the rack-and-pinion gear 2 (Fig. 8) according to the object central axis. Finally, the contact along the active surface is assured with the leaf springs pushing the bowl 3 [11], so the linear contact with the finger 4 is permanently. As we have mentioned above, the camera 3 has been used for tacking the pictures. The detailed picture of the contact surface is presented in Figure 9. The influence of material elasticity was reduced by using the described mechatronic system. The improvements regarding the grasping of objects with external irregular shapes were focused on two main directions: the increasing design accuracy of the working surfaces based on image taken from the object; the aim of centering the object between the two fingers along an imposed direction keeping the contact in as many points as possible. The experimental set-up pointed out the solution for keeping the contact permanently between the finger and the object.

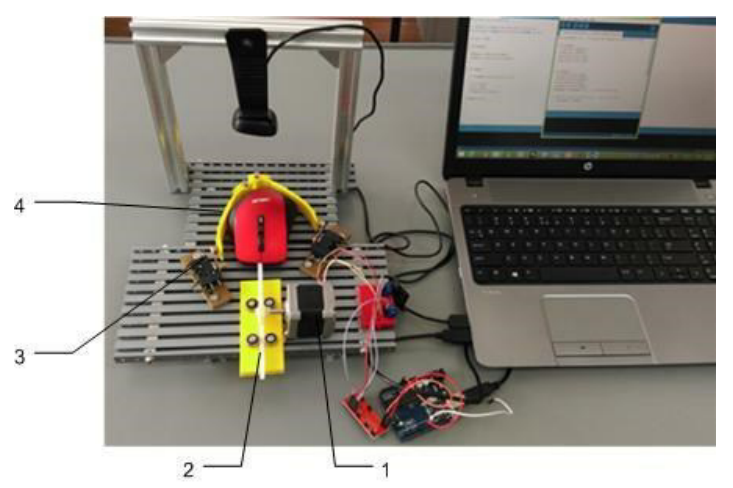

Fig. 8. The experimental set-up. 


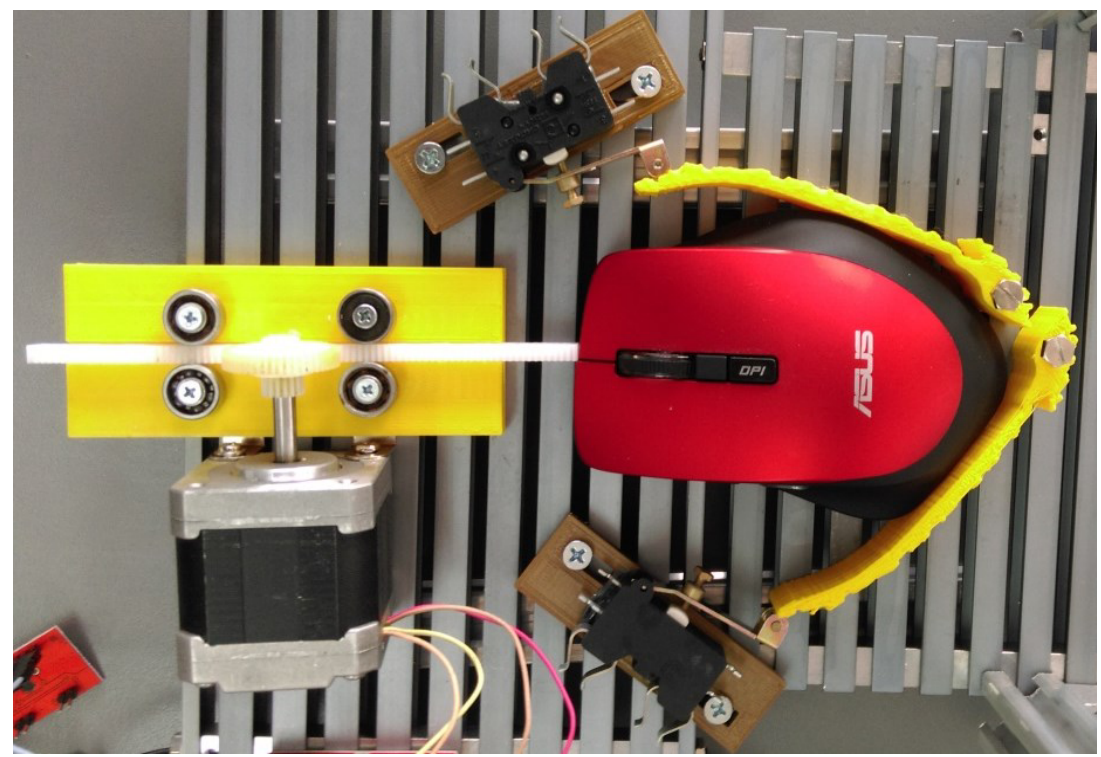

Fig. 9. The contact between the object and the fingers.

As future work, we aim to study the elasticity of the finger during the grasping taking into account the described solution for keeping the contact with the object.

\section{Conclusions}

The paper aims to present the method of image processing in order to be used for designing the gripper used for grasping the objects with irregular shapes. Meantime, the mechatronic system for improving and maintaining the contact points has been described. There are many algorithms applied for image processing, so we have chosen the Matlab specialized functions with some specific parameters depending on the object dimensions. The method should be particularized for each object, which implies an increasing price, being a disadvantage.

The mathematical computation provided the position of each contact point. Based on them, the active surface has been three-dimensional modeled. Two fingers were manufactured applying the rapid prototyping technique. The mechatronic system was designed in order to assure the permanently contact between the fingers and the object.

As future work, we should study the influence of material elasticity and the grasping stability during the dynamic process.

\section{References}

1. D. Morrison, P. Corke, J. Leitner, Closing the Loop for Robotic Grasping: A Real-time, Generative Grasp Synthesis Approach, Robotics:Science and Systems, Pittsburgh, PA (2018)

2. H. Dong, G. Sun, W Pang, E. Asadi, Fast Ellipse Detection via Gradient Information for Robotic Manipulation of Cylindrical Objects, IEEE Robotics and Automation Letters, 3, 4, pp. 2754 $2761(2018)$

3. F. Husain, A. Colome, B. Dellen, G. Alenya, G. Torras, Real-time tracking and grasping of a moving object from range video, IEEE International Conference on Robotics and Automation (ICRA) (2014)

4. D. Markovic, D. Vujicic, D. Mitrovic, S. Randic, Image Processing on Raspberry Pi Cluster, International Journal of Electrical Engineering and Computing, 2, 2, pp. 83-90 (2018) 
5. A. Haj-Ali, R.B. Hur, N. Wald, R. Ronen, S. Kvatinsky, Imaging: In-memory Algorithms for Image Processing, IEEE Transaction on Circuits and Systems-I, 65, 12, pp. 4258 - 4271 (2018)

6. E. P. Petrov, N. L. Kharina, Markov Processes in Image Processing, International Conference Information Technologies in Business and Industry, IOP Conf. Series 1015 (2018)

7. S. Khedaskar, M.A. Rokade, B. Patil, P.N.Tatwadarshi, A Survey of Image Processing and Identification Techniques, Viva-Tech International Journal for Research and Innovation, 1, 1, pp. $1-10(2018)$

8. A. McAndrew, Introduction to Digital Image Processing with Matlab, ISBN-13: 978$0534400118(2014)$

9. M. Sinecen, Digital Image Processing with MATLAB, Applications from Engineering with Matlab Concepts, Chapter Digital Image processing with Matlab (2016)

10. E. Moraru, O. Dontu, A. Petre, D. Vaireanu, F. Constantinescu, D. Besnea, Some technological particularities on the execution of dental prostheses realized by selective laser deposition, Journal of Optoelectronics and Advanced Material, 20, Issue 3-4, pp. 208-213 (2018)

11. D. Besnea, C. Rizescu, D. Rizescu, D. Comeaga, R. Ciobanu, E. Moraru, Study of deflection behavior of 3D printed leaf springs IOP Conference Series: Materials Science and Engineering, 444, Issue 4 (2018) 